\title{
A COMPACT INDUSTRIAL HIGH-CURRENT CONTINUOUS WAVE ELECTRON LINAC
}

\author{
$\underline{\text { A.S. Alimov }}^{* \dagger}$, D.I. Ermakov ${ }^{*}$, B.S. Ishkhanov ${ }^{*}$, E.A. Knapp, A.F. Salakhutdinov ${ }^{*}$, \\ V.I. Shvedunov, and W.P. Trower, World Physics Technologies, Blacksburg VA
}

\begin{abstract}

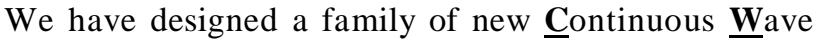
$\underline{\text { LINear }} \underline{\mathbf{A C}}$ celerators for electron-based industrial, medical, and environmental irradiation applications. Our ten reliable, small, inexpensive high-power modular accelerators will produce beams with energies from 0.6 to $6.0 \mathrm{MeV}$ in increments of 600 $\mathrm{keV}$, each with a current selectable from 0 to $50 \mathrm{~mA}$. We have constructed the critical gun- ${ }^{\text {st }}$ section model, which has undergone the first beam test. We have achieved beam parameters of $600 \mathrm{keV}, 10 \mathrm{~mA}$, and 6 $\mathrm{kW}$ and we have demonstrated all the innovations of our initial design [1].
\end{abstract}

\section{INTRODUCTION}

Particle accelerators with increasing beam power are being required for large-scale industrial production lines, as well as for the destruction of biological and chemical waste. Although direct current machines produce adequate beams of $\sim 1 \mathrm{MeV}$, raising the beam energy significantly increases their size, weight, and cost. Thus, we are constructing a family of ten accelerators to produce electrons with energies from 0.6 to $6 \mathrm{MeV}$ in increments of $600 \mathrm{keV}$ with corresponding beam power from 30 to $300 \mathrm{~kW}$. Only our $1^{\text {st }}$ accelerating section is a non-standard section since it must accelerate $15 \mathrm{keV}$ gun electrons to a relativistic energy of $600 \mathrm{keV}$. Subsequent identical sections will each increase the beam energy by 600 $\mathrm{keV}$.

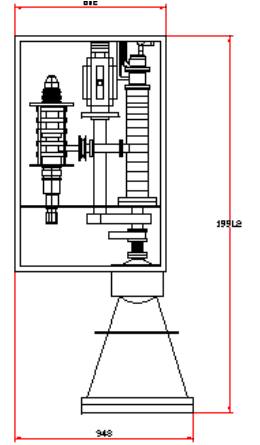

Figure 1: Gun- $1^{\text {st }}$ section LINAC schematic

Having completed the design of our accelerator family, we are commissioning a prototype gun- $1^{\text {st }}$ section model which uses a: (1) Low-energy $15 \mathrm{keV}$ thermionic electron gun joined directly to the first Accelerating Structure cell; (2) High capture efficiency for low-energy gun electrons AS; (3) Simple reliable $\underline{\text { Radio }}$ Frequency power supply system; and (4) Common gun-klystron power supply. Our LINAC is shown in Fig. 1 and its principal parameters are listed in Table 1.

\begin{tabular}{ll}
\multicolumn{2}{c}{ Table 1: Gun- $1^{\text {st }}$ section parameters } \\
\hline Output beam energy & $0.6 \mathrm{MeV}$ \\
Beam current & 0 to $50 \mathrm{~mA}$ \\
Maximum beam power & $30 \mathrm{~kW}$ \\
Length & $1.2 \mathrm{~m}$ \\
Weight & $\sim 70 \mathrm{~kg}$ \\
Gun/klystron $\quad$ high & $15 \mathrm{kV}$ \\
voltage & \\
Plug power consumption & $\sim 75 \mathrm{~kW}$ \\
Electric efficiency & $\sim 40 \%$ \\
\hline
\end{tabular}

\section{ELECTRON GUN}

Since we have used a common power supply for the gun and the klystron, our electron gun beam energy is $15 \mathrm{keV}$. To make our accelerators compact, we eliminated the traditional bunching system, drift space, and focusing elements and mount the gun directly to the AS. The gun currents are selectable from 0 to $250 \mathrm{~mA}$ with $100 \mathrm{~mA}$ being the nominal operating current. The gun beam radii are less than $2.5 \mathrm{~mm}$ with crossovers at greater than $50 \mathrm{~mm}$ from the cathode so that the beam is convergent in the first AS cell.

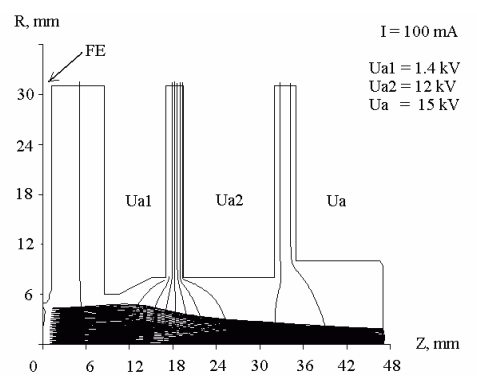

Figure 2: Gun schematic with beam trajectories [2]

We satisfied these requirements with a three-anode gun, shown in Fig. 2, that has a $8.6 \mathrm{~mm}$ diameter spherically concave cathode. The Focusing Electrode is at the cathode potential while the two intermediate anodes are held at Ua1 and Ua2 and the main anode,

\footnotetext{
* Permanent address: Institute of Nuclear Physics, Moscow State

University, Moscow, Russia.

† Email: wptinc@naxs.net
} 
located at the gun exit, is held at $\mathrm{Ua}=15 \mathrm{kV}$. The intermediate anode potentials are defined by $\mathrm{Ua1}=$ $\mathrm{k} \cdot 1.75 \mathrm{kV}$ and $\mathrm{Ua} 2=\mathrm{k} \cdot \mathrm{Ua}$, where the coefficient, $0 \leq \mathrm{k}$ $\leq 1$, provides current regulation from zero $(\mathrm{k}=0)$ to $250 \mathrm{~mA}(\mathrm{k}=1)$. Once manufactured, we tested our gun, as seen in Fig. 3, with currents up to $50 \mathrm{~mA}$ before installing it on the AS.

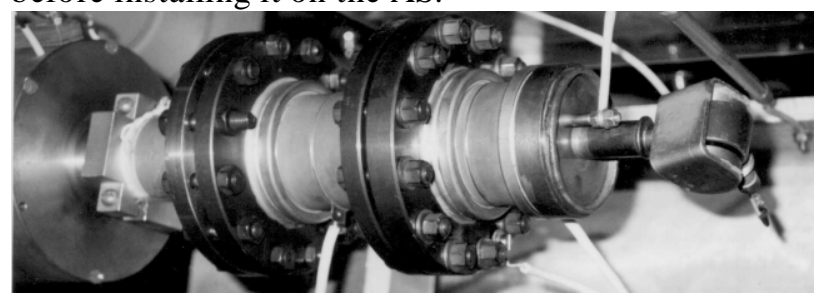

Figure 3: Gun at the test stand

\section{BEAM DYNAMICS}

The $1^{\text {st }}$ AS has 14 cells with $\beta$ from 0.237 to 0.888 . Injecting a $15 \mathrm{keV}$ direct current gun beam into the initial AS cells, which must then form bunches with energy up to $\sim 75 \mathrm{keV}$, places large demands on the beam dynamics. We designed the subsequent $\beta>0.5$ cells by roughly scaling the cells with $\beta=1$. We calculated the beam dynamics [3] principally to optimize the longitudinal and transverse beam motion in the first AS cells and then to further optimize the entire AS beam dynamics using "real" electron gun beam parameters and space charge effects.

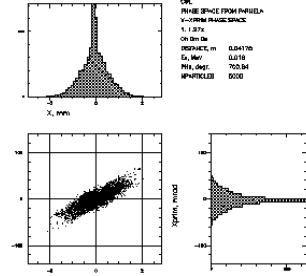

(a)

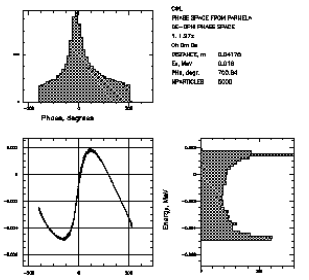

(b)
Figure 4: Second AS cell center phase space: (a) transverse and (b) longitudinal

We must provide high capture efficiency, $I_{\text {ou }} / I_{\text {gun }}$, in the initial AS cell that is acting as a pre-buncher. To accomplish this we must provide the maximum bunching parameter (i.e., the maximum first current harmonic) at the second cell (the first accelerating cell) center. The transverse and longitudinal beam phase spaces at the $2^{\text {nd }}$ cell center, shown in Fig. 4, confirm the pre-buncher cell effectiveness by the tightness of the longitudinal phase space.

\begin{tabular}{cl} 
Table 2: Gun- ${ }^{\text {st }}$ & section beam parameters \\
\hline $\mathrm{I}_{\text {gun }}$ & $100 \mathrm{~mA}$ \\
$\mathrm{I}_{\text {out }}$ & $51 \mathrm{~mA}$ \\
$\left\langle\mathrm{~W}_{\text {beam }}\right\rangle$ & $610 \mathrm{keV}$ \\
$\Delta \mathrm{W}_{\text {beam }}$ & $\pm 20 \mathrm{keV}$ \\
$\Delta \varphi_{\text {beam }}$ & $\sim 50 \mathrm{deg}$ \\
Norm. $\left\langle\varepsilon_{\mathrm{x}}\right\rangle$ & $27.5 \mathrm{~mm} \times \mathrm{mrad}$ \\
Norm. $\left\langle\varepsilon_{\mathrm{y}}\right\rangle$ & $28.3 \mathrm{~mm} \times \mathrm{mrad}$ \\
\hline
\end{tabular}

The AS transverse and longitudinal output beam phase spaces are shown in Fig. 5 and the output beam parameters are given in Table 2 .

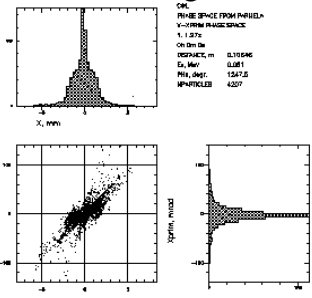

(a)

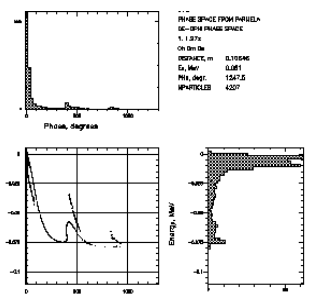

(b)
Figure 5: AS output phase space: (a) transverse and (b) longitudinal

\section{ACCELERATING STRUCTURE}

Initially we separately optimized the three low $\beta$ cells and then the higher $\beta$ cells. The pre-buncher cell has $1 / 9$ the on-axis field amplitude of the first accelerating cell. We used the preliminary beam dynamics results to calculate the first two cells, shown in Fig. 6, whose gap lengths and distances were unchanged in the optimization.

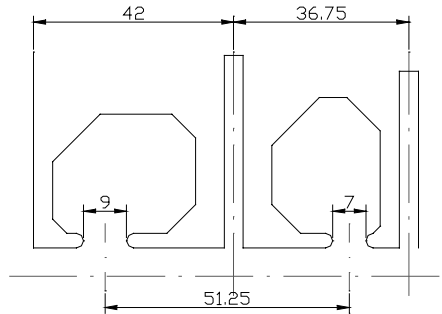

Figure 6: Pre-buncher and first accelerating cell geometry

We obtained the required on-axis AS field amplitude ratio with a $56^{\circ}$ pre-buncher cell coupling slot and a $24^{\circ}$ first accelerating cell coupling slot. After optimizing the coupling slot size and position and cell radii, we tuned the frequency and field ratio of the first two accelerating cells by scaling the prebuncher, first coupling cell, and first accelerating cell dimensions. We then varied the second accelerating cell radius. Figure 7(a) shows the initial three AS cells in a mesh representation, while Fig. 7(b) shows the onaxis longitudinal electric field distribution.

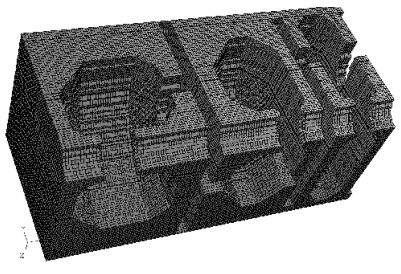

(a)

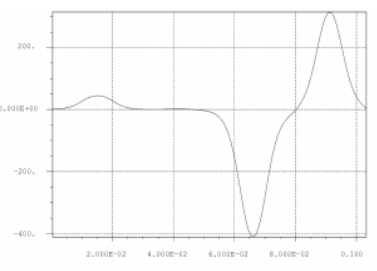

(b)
Figure 7: First AS cell (a) mesh and (b) $E_{\mathrm{z}}(0,0, \mathrm{z})$

Next we optimized and tuned a $\beta=1$ cell to obtain a $5 \%$ coupling and an $88 \mathrm{M} \Omega / \mathrm{m}$ effective shunt impedance. RF losses to the structure wall were $\sim 1.070$ 
$\mathrm{kW}$ per cell for a $1 \mathrm{MeV} / \mathrm{m}$ accelerating gradient. We placed the coupling slots far off-axis to avoid overheating the cell noses and our large slots were beneficial when we pumped the structure. The accelerating cell coupling slot orientation was chosen to compensate for focusing in the adjacent accelerating and coupling cells. The coupling cells slot orientation was rotated by $90^{\circ}$ relative to the accelerating cells. Finally, we manufactured, tuned, and brazed the AS and measured the on-axis $E_{z}$-field distribution, seen in Fig. 8, which agrees fairly well with our calculations.

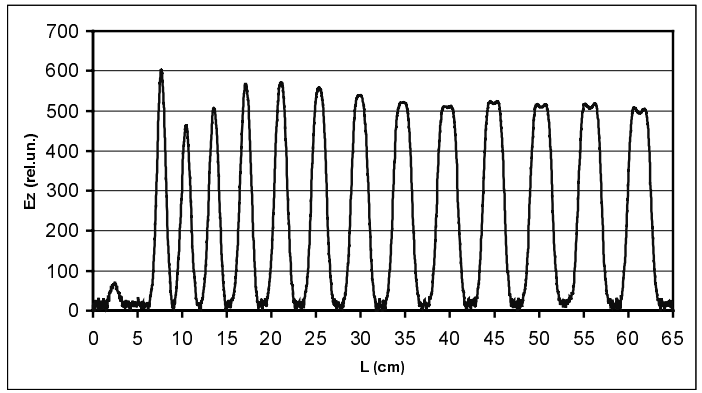

Figure 8: Measured on-axis longitudinal field

\section{RADIO FREQUENCY SYSTEM}

Our simple reliable RF system, seen in Fig. 9, uses self-excitation in a positive klystron-section feedback loop whose reliability we demonstrated experimentally with our previous prototype accelerator [1].

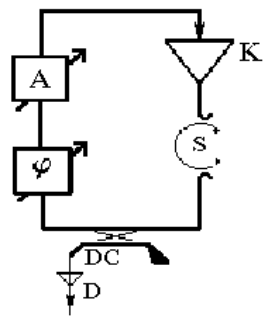

Figure 9: RF system schematic

We used a $50 \mathrm{~kW} \mathrm{CW} \mathrm{klystron} \mathrm{(K)} \mathrm{to} \mathrm{drive} \mathrm{the} \mathrm{AS.}$ Some $15 \mathrm{~kW}$ of the klystron power is dissipated in the structure walls providing the accelerating field and, depending on beam current, up to $30 \mathrm{~kW}$ goes into the beam. When operating in the self-excited mode, the system oscillates at the structure resonant frequency, which the klystron frequency automatically follows. A RF probe provides the structure signal that passes through the electrically driven coaxial phase-shifter $(\varphi)$ and $p$-i-n-attenuator (A), and then enters the klystron. The self-excitation phase conditions are chosen by the phase-shifter while the feedback p-i-n attenuator regulates the klystron output power and, consequently, the accelerating field amplitude. This amplitude is controlled by a diode (D) whose signal is used by the amplitude stabilization system that controls the p-i-n attenuator current. Thus our accelerating field amplitude is stable to $\sim 0.001$, which is essential for our high beam loading.

We eliminated the traditional circulator by coupling the klystron directly to the AS and by operating in the self-excited mode. We tuned the system by determining the klystron body current dependence on the connecting wave-guide phase length. With the minimum body current, we found the length that minimizes the reflected RF power influence on the output klystron cavity. Thus, with no circulator, we reduced the accelerator size, weight, and cost. Our high power AS test set-up, seen in Fig. 10 with its circulator-less klystron system, operated stably for several hundred hours without component failures.

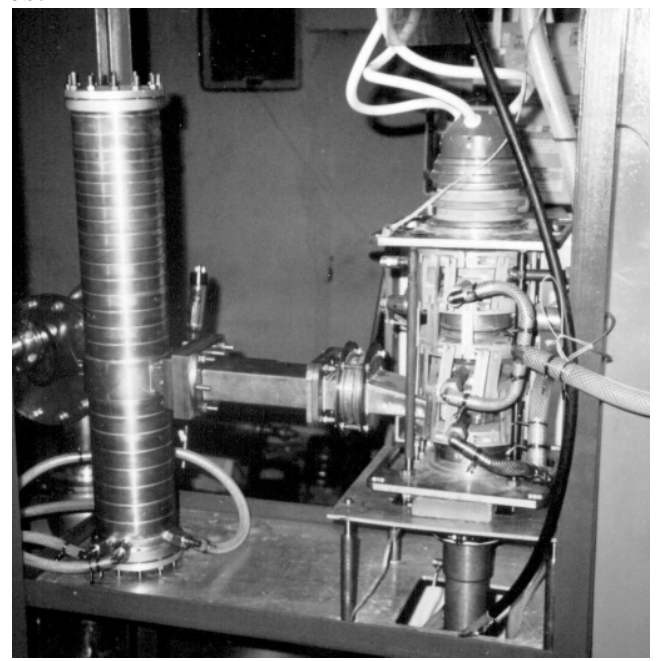

Figure 10: High-power AS tests

\section{CONCLUSION}

We have constructed the simplest of our new family of industrial CW LINACs, thereby validating our long held design ideas. In the first beam tests, our single section model has provided a $600 \mathrm{keV}, 10 \mathrm{~mA}, 6 \mathrm{~kW}$ electron beam at a $20 \mathrm{~mA}$ gun current, thus demonstrating a design $50 \%$ capture efficiency and beam energy. The accelerator tests are now in progress. Increasing the beam current to $50 \mathrm{~mA}$ will require thorough electron gun training and modification of the Faraday Cup and vacuum system to improve the vacuum conditions in the accelerator.

\section{REFERENCES}

[1] A.S. Alimov, A.S. Chepurnov, O.V. Chubarov, D.I. Ermakov, B.S. Ishkhanov, K.A. Gudkov, I.M. Piskarev, V.I. Shvedunov, and A.V. Shumakov, "Experimental Investigation of a CW Electron Linac with High Beam Current", Instrum. Exp. Tech. 37 (1994) 7; O.V. Chubarov, A.S. Alimov, and V.I. Shvedunov, "A Compact Industrial CW LINAC", IEEE Trans. Nucl. Sci. 44 (1997) 1037; and A.S. Alimov, O.V. Chubarov, and V.I. Shvedunov, "A HighPower LINAC Power Supply”, IEEE Trans. Nucl. Sci. 44 (1997) 1033.

[2] W.B. Herrmannsfeld, "EGUN an Electron Optics and Gun Design Program", SLAC-Report-331, 1988.

[3] PARMELA code originally developed by K.R. Crandall. 